\title{
Surgical resection using retroperitoneal approach for solitary fibrous tumor in the pelvis
}

\author{
SUNAO SHOJI $^{1}$, MAYURA NAKANO ${ }^{1}$, SHINYA YAMAMOTO $^{2}$, NAOTAKA OKADA $^{3}$, YOSHIHIRO NAGATA ${ }^{1}$, \\ YASUTOMO SEKIDO ${ }^{4}$, HAYATO TERAYAMA ${ }^{5}$, MASAHIRO ITO ${ }^{5}$ and TOYOAKI UCHIDA ${ }^{1}$ \\ ${ }^{1}$ Department of Urology, Tokai University Hachioji Hospital; ${ }^{2}$ Department of Urology, The Cancer Institute Hospital of \\ Japanese Foundation for Cancer Research; ${ }^{3}$ Department of Surgery, Tokai University Hachioji Hospital; ${ }^{4}$ Department of \\ Pathology, Tokai University Hachioji Hospital; ${ }^{5}$ Department of Anatomy, Tokyo Medical University, Tokyo, Japan
}

Received January 13, 2011; Accepted April 26, 2011

DOI: $10.3892 / \mathrm{ol} .2011 .314$

\begin{abstract}
This report concerns a case of solitary fibrous tumor (SFT) for which surgical resection was performed using a retroperitoneal approach. A 41-year-old man was referred to our hospital with urinary retention. Abdominal ultrasound sonography (US) and computed tomography (CT) showed a hypervascular mass lesion in the pelvis. Transrectal biopsy showed SFT. Surgical resection was carried out using a retroperitoneal approach and preserving the neural network related to urinary and erectile functions. Based on immunohistochemical findings, the tumor was diagnosed to be malignant SFT in the pelvic cavity. Urinary function improved post-operation. There was no change to IIEF-5 and it continued to function well. The patient showed no sign of recurrence 12 months after surgery and required no additional therapy.
\end{abstract}

\section{Introduction}

SFTs, which are known to be either localized fibrous tumors or fibrous mesotheliomas, are rare spindle-cell neoplasms first reported as originating in the pleura (1). Although most reported SFTs tend to originate within the thoracic cavity, extrathoracic SFTs, which occur in a wide range of anatomic sites, have also been reported (2). Recent immunohistochemical and ultrastructural studies support the mesenchymal histogenesis of SFT (3). Most SFTs in deep soft tissue are indolent and are found incidentally in the course of examinations for other disorders or during medical check-ups. Some cases are also diagnosed with hypoglycemia, due to insulin-like growth factor II (IGF-2) secreted by the tumor cells of SFT (4). In the present case, SFT was found in the pelvis, with urinary retention caused by tumor pressure on the urethra.

Correspondence to: Dr Sunao Shoji, Department of Urology, Tokai University Hachioji Hospital, Ishikawa-machi 1838, Hachioji, Tokyo 192-0032, Japan

E-mail: sunashoj@mail.goo.ne.jp

Key words: solitary fibrous tumor, surgical resection
SFT with high cellularity, pleomorphism and increased mitotic activity is classified as malignant SFT. However, infiltrative features that prevent total resection significantly affect their outcome. The outcome for patients with extrapleural SFTs is unpredictable based on histological assessment (5). Therefore, complete resection is essential to prolong prognosis. However, surgical resection of a large tumor in the pelvis often injures the pelvic nerve plexus and damages urinary and erectile functions. In this study, we attempted complete surgical resection while preserving urinary and erectile functions.

\section{Case report}

A 41-year-old man was referred to our hospital with urinary retention. Abdominal ultrasound sonography (US) and computed tomography (CT) revealed a hypervascular mass lesion, measuring $125 \times 95 \times 129 \mathrm{~mm}$, in the pelvis (Fig. 1). Magnetic resonance imaging (MRI) showed a mass with low signal intensity on T1-weighted images and a high signal intensity on T2-weighted images, as well as mass-oppressed bladder, prostate and rectum in the retroperitoneum. Blood biochemistry and urine examination results were in the normal ranges, including prostate-specific antigen (PSA), $\alpha$-fetoprotein (AFP), carcinoembryonic antigen (CEA) and carbohydrate antigen 19-9 (CA19-9). Transrectal biopsy showed SFT.

Following the diagnosis of SFT, a surgical resection was performed using a retroperitoneal approach to prevent peritoneal defects. During surgery, we performed an abruption between the bladder and the peritoneum, entered the retroperitoneum, and confirmed that the tumor was not connected to neighboring organs such as the bladder, prostate, seminal vesicle or rectum. A complete resection was performed, while preserving the neural network related to urinary and erectile function, such as the bladder branch of the pelvic nerve plexus and neurovascular bundles (NVB). Macroscopically, the resected tumor was well circumscribed and encapsulated, measuring 120x92x115 mm and weighing 2,200 gr. The cut surface was grayish-white, showing components of hemorrhaging. Microscopically, the tumor was composed of spindle-shaped and oval cells in sarcoma, based on moderate mitotic rate and cellularity (Fig. 2). Immunohistochemically, the tumor was positive for CD34, CD99, vimentin, bcl-2 and 


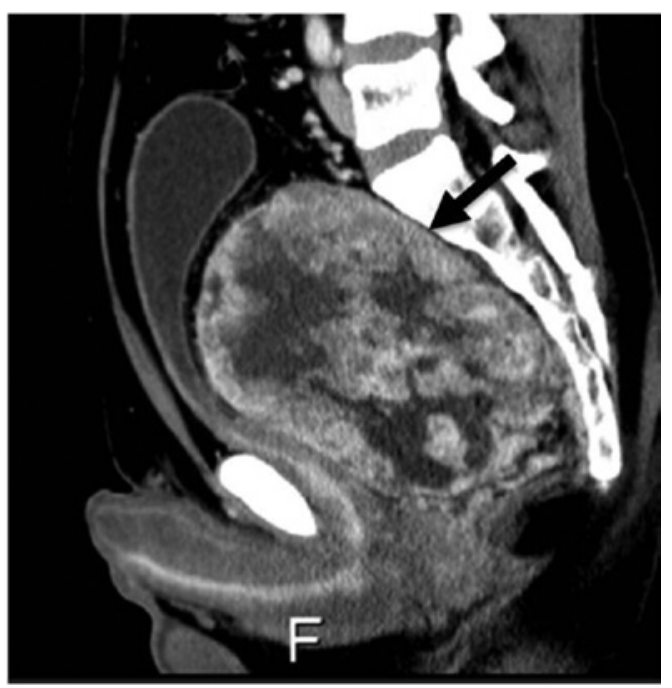

Figure 1. Computed tomography revealed a hypervascular mass lesion, measuring $125 \times 95 \times 129 \mathrm{~mm}$, in the pelvis $(\rightarrow)$.

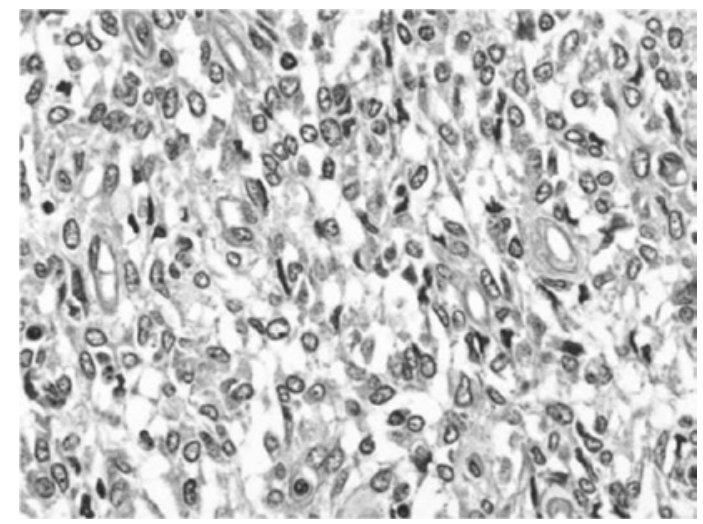

Figure 2. Microscopically, the tumor was composed of spindle-shaped and ovoid cells in sarcoma, based on a moderate mitotic rate and cellularity.

p53, but was negative for keratin, $\alpha$-smooth muscle actin and S100 protein. The MIB-1 index, which indicates cell proliferation capability, was 5\%. Based on these findings, the tumor was diagnosed to be malignant SFT in the pelvic cavity. Prior to the operation, the scores of IPSS, QOL index, OABSS, and IIEF-5 were 19, 6, 11 and 14, respectively. Six months after the operation the scores had changed to $1,0,1$ and 13 , respectively. Moreover, max flow rate, voiding time and residual urine were $18 \mathrm{ml} / \mathrm{sec}, 19 \mathrm{sec}$ and $35 \mathrm{ml}$. The patient showed no sign of recurrence 12 months after surgery and required no additional therapy.

\section{Discussion}

Since the initial description that SFT originates in the pleura, SFTs have been reported to occur in a wide range of anatomic sites $(1,2)$. Among extrathoracic SFTs, primary SFT in the pelvic cavity occurred in $16 \%$ of 79 cases of SFTs involving various sites (2). The key symptoms of abdominal SFTs are abdominal pain, abdominal fullness, or a palpable abdominal mass. Hypoglycemia occurred in $4 \%$ of 360 cases of SFTs

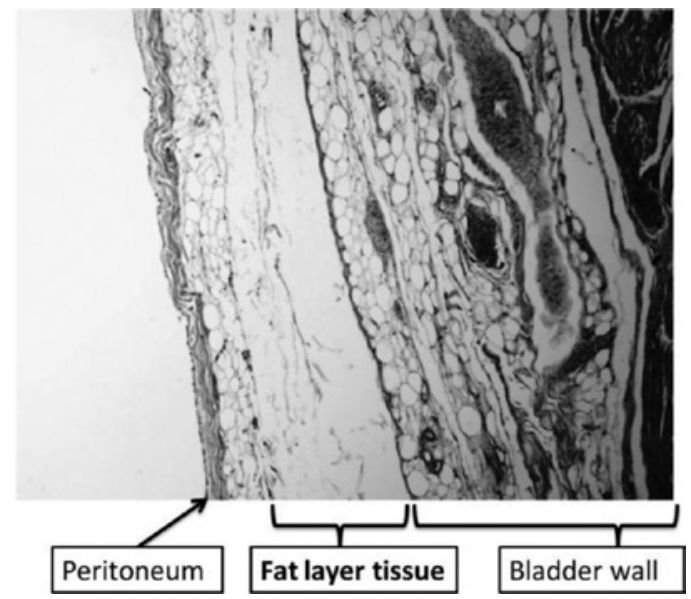

Figure 3. In the anatomical study, a fat layer tissue was revealed between the peritoneum and bladder wall.

of the pleura (6). In the present case, no hypoglycemia was detected. The symptom of the present case was urinary retention caused by tumor pressure on the urethra. However, no previous cases of SFT with the symptom of urinary retention were found in the literature. CT imaging of SFT usually revealed a well-delineated, homogeneous, and occasionally lobulated mass of soft tissue attenuation (7), and MRI revealed fibrous tissue of SFT with a low signal intensity on T1-weighted images. However, mature fibrous tissue has a low intensity, while malignant fibrous tissue tends to exhibit a high signal intensity on T2-weighted images (4). Although the radiological findings in our case were typical of SFT, we performed US-guided needle biopsy for diagnosis since cases of SFT are rare. The pathological features of SFT were a 'patternless pattern' characterized by a haphazard, storiform arrangement of short spindle or ovoid cells, and a 'hemangiopericytoma-like appearance' with prominent vascularity by thin-walled vessels (8).

Immunohistochemical examination of proteins, such as CD34 and bcl-2, has been found to be helpful for diagnosing SFT (8). In their study, England et al reported that more than one-third of pleural SFTs are histologically malignant, and proposed pathological criteria for malignant SFT such as high cellularity with crowded and overlapping nuclei and over 4 mitotic figures per 10 high-power fields. However, the clinical features of retroperitoneal SFT are not necessarily concordant with morphologic evaluations, and most extrapleural SFTs in previous reports, even with malignant histological features, showed a benign nature. Some investigators stressed that intraoperative findings and surgical resectability are more reliable prognostic factors based on the experience of a small number of patients with recurrent or metastasized SFT (9). SFTs have a low rate of local recurrence and metastasis following surgical resection. However, tumors larger than $10 \mathrm{~cm}$ or those demonstrating a histologically malignant component have an increased risk of local recurrence and metastasis. Complete en bloc surgical resection is the standard therapy for SFT. We performed complete resection of the tumor without any adhesion, and the pathological finding of margin was negative for tumor cells in the present case. In addition, surrounding tissue and peritoneum was 
not damaged when resecting the pelvic mass, preserving the neural network connected to urinary and erectile functions in the pelvic cavity. Urinary function improved and erectile function remained good following the operation. In the anatomical study, a fat layer tissue was revealed between the peritoneum and bladder wall (Fig. 3). Consequently, we were able to perform abruption between the bladder and peritoneum and enter the retroperitoneum. Takizawa et al reported that retroperitoneal SFTs required less adhesion than tumors of other histotypes at the same location, including liposarcoma, leiomyomas, leiomyosarcomas, malignant fibrous histiocytomas, nerve sheath tumors, and germ cell tumors (10). We propose that pelvic SFTs detected with no adhesion in and around the tumor can be completely resected with ease, while preserving the neural network connected to urinary and erectile functions in the pelvic cavity.

\section{References}

1. Klemperer P and Rabin CB: Primary neoplasm of the pleura: a report of five cases. Arch Pathol 11: 385-412, 1931.

2. Gold JS, Antonescu CR, Hajdu C, et al: Clinicopathological correlates of solitary fibrous tumors. Cancer 94: 1057-1068, 2002.
3. Suster S, Nascimento AG, Miettinen M, et al: Solitary fibrous tumors of soft tissue. A clinicopathological and immunohistochemical study of 12 cases. Am J Surg Pathol 19: 1257-1266, 1995.

4. Nagase T, Adachi I, Yamada T, et al: Solitary fibrous tumor in the pelvic cavity with hypoglycemia: report of a case. Surg Today 35: 181-184, 2005.

5. England DM, Hochholzer L and McCarthy MJ: Localized benign and malignant fibrous tumors of the pleura. A clinicopathologic review of 223 cases. Am J Surg Pathol 13: 640-658, 1989.

6. Briselli M, Mark EJ and Dickersin GR: Solitary fibrosis tumors of the pleura: eight new cases and review of 360 cases in the literature. Cancer 47: 2678-2689, 1981.

7. Lee KS, Im JG, Choe KO, Kim CJ and Lee BH: CT findings in benign fibrous mesothelioma of the pleura. Am J Roentgenol 158: 983-986, 1992

8. Yamashita S, Tochigi T, Kawamura S, et al: Case of retroperitoneal solitary fibrous tumor. Hinyoukika Kiyo 53: 477-480, 2007.

9. Morimitsu Y, Nakajima M, Hisaoka M, et al: Extrapleural solitary fibrous tumor: clinicopathologic study of 17 cases and molecular analysis of the p53 pathway. Acta Pathol Microbiol Immunol Scand Suppl 108: 617-625, 2000.

10. Takizawa I, Saito T, Kitamura Y, et al: Primary solitary fibrous tumor (SFT) in the peritoneum. Urol Oncol 26: 254-259, 2008. 